Pacific Journal of Mathematics

A GEOMETRIC BOUND FOR MAXIMAL FUNCTIONS

DETLEF MÜLLER 


\section{A GEOMETRIC BOUND FOR MAXIMAL FUNCTIONS ASSOCIATED TO CONVEX BODIES}

\section{DetLef MÜlleR}

For a convex symmetric body $B$ in $\mathbb{R}^{n}$ let $M_{B}$ denote the centered maximal operator

$$
M_{B} f(x)=\sup _{t>0} \frac{1}{\operatorname{Vol} B} \int|f(x+t y)| d y
$$

for $f \in L_{\text {loc }}^{1}\left(\mathbb{R}^{n}\right)$. We associate with $B$ two linear invariants $\sigma(B)$ and $Q(B)$, and show that for $p>1$ the norm of the operator $M_{B}$ on $L^{p}\left(\mathbb{R}^{n}\right)$ is bounded by a constant which may depend on $p, \sigma(B)$ and $Q(B)$, but not explicitly on the dimension $n$. In particular, if $B_{q}$ denotes the unit ball in $\mathbb{R}^{n}$ with respect to the $l^{q}$-norm, we can prove that $M_{B_{q}}$ has a bound on $L^{p}\left(\mathbb{R}^{n}\right)$ which is independent of $n$, provided that $1 \leq q<\infty$.

The behaviour of maximal functions associated to convex bodies has been studied by various authors during recent years. When $B$ is the Euclidean ball, i.e. $B=B_{2}$, Stein [9] has shown that $M_{B}$ is bounded on $L^{p}\left(\mathbb{R}^{n}\right)$ uniformly in $n$ for every $p>1$, and Bourgain [2, $3,4]$ and Carbery [6] have shown that the analogue of this holds for any convex body $B$, provided $p>3 / 2$. Moreover, by a result of Stein and Strömberg [11] it is known that the $L^{p}$ operator norm $\left\|M_{B}\right\|_{p, p}$ of $M_{B}$ grows at most linearly in the dimension $n$ for any $p>1$.

Since the general estimates for convex bodies in [2] do not imply that $\left\|M_{B}\right\|_{p, p}$ has a bound independent of $n$, if $p \leq 3 / 2$, it is well possible that for $p \leq 3 / 2$ one can only hope for estimates of $\left\|M_{B}\right\|_{p, p}$ which depend on additional geometric invariants associated with the body $B$. In this article, we shall show that one can in fact prove an estimate of this kind:

We associate with $B$ the following two linear invariants $\sigma(B)$ and $Q(B)$ : There exists a regular linear transformation $S$ of $\mathbb{R}^{n}$, which is unique modulo orthogonal transformations, and a unique constant $L(B)$ such that $\operatorname{Vol}_{n} S(B)=1$ and

$$
\int_{S(B)}|\langle x, \xi\rangle|^{2} d x=L(B)^{2}
$$

for all unit vectors $\xi \in \mathbb{R}^{n}$. Let $1 / \sigma(B)$ be the minimum of all $(n-1)$ dimensional volumes of all sections of $S(B)$ by hyperplanes, and $Q(B)$ 
the maximum of the $(n-1)$-dimensional volumes of all orthogonal projections of $S(B)$ onto hyperplanes (we note that $\sigma(B) \approx L(B)$ ). Then, for $p>1$, the operator norm $\left\|M_{B}\right\|_{p, p}$ can be estimated by a constant depending only on $p, \sigma(B)$ and $Q(B)$.

This criterion suffices for example to prove the uniform boundedness in $n$ of the maximal function $M_{B_{q}}$, where $B_{q}$ denotes the unit ball with respect to the $l^{q}$-norm on $\mathbb{R}^{n}, 1 \leq q<\infty$. This extends a result of Bourgain [4] who proved it for $q \in 2 \mathbb{N}$ by making use of an "extra" decay of the Fourier transform of $\chi_{B_{2 k}}, \chi_{B_{2 k}}$ denoting the characteristic function of $B_{2 k}$. However, this extra decay depends on some "smoothness" of $B_{q}$ for $q \in 2 \mathbb{N}$, which can easily be destroyed by cutting off a small piece of $B_{2 k}$ along an affine hyperplane, whereas our result is invariant under such operations.

Moreover, since one can show that $Q\left(B_{\infty}\right)=\sqrt{n}$, this might indicate that the norm of the "cubic" maximal operator $M_{B_{\infty}}$ associated with the unit cube of $L^{p}$ is possibly growing with the dimension, if $p \leq 3 / 2$, and our results give some hints how one might try to prove this.

I would like to express my gratitude to the Mathematical Sciences Research Institute in Berkeley for the warm hospitality during my stay there by which this paper was completed, and especially to E. M. Stein for hints concerning multipliers of Laplace-transform type.

2. The main theorem. Let $B$ be a convex symmetric body in $\mathbb{R}^{n}$. Arguing as in [2], we see that there exist a linear transformation $S \in$ $\mathrm{GL}\left(\mathbb{R}^{n}\right)$ and a constant $L(B)>0$ such that

$$
\operatorname{Vol}_{n} S(B)=1 \text { and } \int_{S(B)}|\langle x, \xi\rangle|^{2} d x=L(B)^{2}
$$

for all unit vectors $\xi \in S^{n-1}=\left\{\xi \in \mathbb{R}^{n}:|\xi|^{2}=\sum_{j}\left|\xi_{j}\right|^{2}=1\right\}$. It is easy to see that $L(B)$ is determined uniquely by (1), and that $S$ is unique up to multiplication by an orthogonal transformation from the left.

For $\xi \in S^{n-1}$, we define similarly as in [2]

$$
\varphi(u):=\varphi_{\xi}(u):=\operatorname{Vol}_{n-1}(\{x \in S(B):\langle x, \xi\rangle=u\}), \quad u \in \mathbb{R} .
$$

Moreover, let $\pi_{\xi}$ denote the orthogonal projection of $\mathbb{R}^{n}$ onto the hyperplane perpendicular to $\xi$. Then the constants

$$
\begin{aligned}
1 / \sigma(B) & :=\max \left\{\varphi_{\xi}(0): \xi \in S^{n-1}\right\}, \\
Q(B) & :=\max \left\{\operatorname{Vol}_{n-1}\left(\pi_{\xi}(S(B)): \xi \in S^{n-1}\right\}\right.
\end{aligned}
$$

are obviously linear invariants for $B$, i.e. $\sigma(U(B))=\sigma(B)$ and $Q(U(B))$ $=Q(B)$ for all $U \in \mathrm{GL}\left(\mathbb{R}^{n}\right)$. 
Since also $\left\|M_{B}\right\|_{p, p}$ is a linear invariant for $B$, we therefore may and shall assume in the sequel (except for $\S 3$ ) that $S(B)=B$. Then, by [2], Lemma 1, there exist two universal constants $0<a, A<\infty$, such that

$$
\varphi(u) \leq A \varphi(0) e^{-a \varphi(0)|u|}, \quad u \in \mathbb{R} .
$$

Moreover, there is a universal constant $a_{1}>0$, such that with $L=$ $L(B)$

$$
a_{1}^{-1} \leq L \cdot \varphi_{\xi}(0) \leq a_{1}, \quad \xi \in S^{n-1} .
$$

This implies in particular $\sigma(B) \approx L(B)$.

THEOREM 1. Let $p>1$. Then for all $f \in L^{p}\left(\mathbb{R}^{n}\right)$

$$
\left\|M_{B} f\right\|_{p} \leq C(p, \sigma(B), Q(B))\|f\|_{p},
$$

where the constant $C=C(p, \sigma, Q){ }^{1}$ is independent of $n$ and grows with $\sigma$ and $Q$.

Note that, for $p>3 / 2, C$ can even be chosen to be independent of $\sigma$ and $Q$ by [3] or [6].

Let us fix some notation. We denote by $m$ the multiplier

$$
m(\xi)=\hat{\chi}_{B}(\xi)=\int_{\mathbb{R}^{n}} \chi_{B}(x) e^{-2 \pi i\langle\xi, x\rangle} d x
$$

associated to $\chi_{B}$. If $w \in L^{\infty}\left(\mathbb{R}^{n}\right)$ is any multiplier, we define the corresponding multiplier operator $T_{w}$ as

$$
T_{w}(f)=\mathscr{F}^{-1}(w \hat{f}),
$$

$\mathscr{F}^{-1}$ denoting the inverse Fourier transform.

For $\rho \in \mathbb{R}$ with $\rho>1 / 2$ let us define the $\rho$ th fractional derivative $(\xi \cdot \nabla)^{\rho} m$ of $m$ as in [6] by

$$
\begin{aligned}
(\xi \cdot \nabla)^{\rho} m(\xi) & =\left.\left(\frac{d}{d r}\right)^{\rho}\right|_{r=1} m(r \xi) \\
& =\int(-2 \pi i\langle x, \xi\rangle)^{\rho} K(x) e^{-2 \pi i\langle x, \xi\rangle} d x,
\end{aligned}
$$

where $K=\chi_{B}$. Then, by the results of [6], expecially Theorem 2 and Proposition (ii), our Theorem 1 will be an immediate consequence of

\footnotetext{
${ }^{1}$ Here and in the sequel constants will frequently be denoted by $C$, with the understanding that they may be different from statement to statement.
} 
Proposition 1. Let $1 / 2<\rho<1$. Then for all $f \in L^{p}\left(\mathbb{R}^{n}\right)$

$$
\left\|T_{(\xi \cdot \nabla)^{\rho} m} f\right\|_{p} \leq C_{\rho}(p, \sigma(B), Q(B))\|f\|_{p}
$$

if $1<p<\infty$, where the constant $C_{\rho}$ is again independent of $n$.

This proposition is closely related to the question raised in [6], whether it is possible to find a bound for $T_{(\xi \cdot \nabla) m}$ which is independent of $n$.

The proof of Proposition 1 will be based on analytic interpolation. We define a family of operators $T_{\alpha}=T_{m_{\alpha}}, \alpha \in \mathbb{C}$, by

$$
m_{\alpha}(\xi)=\left.(1+|\xi|)^{1-\alpha}\left[I^{-\alpha} m(r \xi)\right]\right|_{r=1}, \quad \xi \neq 0 .
$$

Here, $I^{-\alpha}$ denotes the $\alpha$ th fractional Riesz derivative with base point 2 , that is

$$
I^{-\alpha} f(r)=\frac{-1}{\Gamma(-\alpha)} \int_{r}^{2}(s-r)^{-\alpha-1} f(s) d s, \quad \operatorname{Re} \alpha<0,
$$

if $\left.\left.f \in C^{\infty}(] 0,2\right]\right)$.

It is well known that $I^{-\alpha}$ can be extended analytically to the whole complex plane, and that $I^{-k}=(d / d r)^{k}$ is the usual $k$ th derivative for $k=0,1, \ldots$. Note that $I^{-\alpha}$ and $(d / d r)^{\alpha}$ as defined in (9) do not agree. However, we shall show later that the difference of these two is unimportant for our problem. We also define $T_{\alpha}^{\varepsilon}=T_{m_{\alpha}^{\varepsilon}}$ by

$$
m_{\alpha}^{\varepsilon}(\xi)=(1+|\xi|)^{-\varepsilon} m_{\alpha}(\xi), \quad \varepsilon>0 .
$$

The proof of Proposition 1 will essentially be contained in the Lemmas 2 and 4 to follow, which deal with the two endpoint cases for the interpolation. Lemmas 1 and 3 are more of a technical nature.

LEMma 1. Let $0 \leq \operatorname{Re} \alpha<1, k \in \mathbb{N}$. Then for $u>1$

$$
\begin{aligned}
& \left|\int_{0}^{u} \frac{s^{-\alpha}}{(1+s / u)^{k}} e^{-2 \pi i s} d s-\frac{e^{\frac{\pi}{2} \alpha i}}{i} \Gamma(1-\alpha)\right| \\
& \quad \leq C_{k} e^{(\pi / 2)|\operatorname{Im} \alpha|} u^{-\operatorname{Re} \alpha} .
\end{aligned}
$$

The proof of Lemma 1 is an easy consequence of Cauchy's integral theorem and follows by changing the path of integration from the interval $[0, u]$ to $-i[0, u]$, connecting those two paths by quarter circles of radii $u$ and $\varepsilon, \varepsilon \rightarrow 0$. We shall omit the technical details. 
Lemma 2. Fix $N>0$ and $0<\varepsilon<1 / 2$. Then

(i) $\left\|m_{\alpha}\right\|_{\infty} \leq C_{N}(\sigma(B), Q(B)) e^{2 \pi|\operatorname{Im} \alpha|}, 0 \leq \operatorname{Re} \alpha<N$,

(ii) $\left\|m_{\alpha}^{\varepsilon}\right\|_{\infty} \leq C_{N}(\sigma(B), Q(B)) e^{2 \pi|\operatorname{Im} \alpha|},-\varepsilon \leq \operatorname{Re} \alpha \leq N$.

Proof. Assume $\operatorname{Re} \alpha \geq-\varepsilon$, and let $k=[\operatorname{Re} \alpha]$ be the integer part of $\operatorname{Re} \alpha$. Then it follows easily by partial integration from (10) that

$$
\begin{aligned}
m_{\alpha}(\xi)= & \left.\sum_{j=0}^{k} \frac{(-1)^{j+1}}{\Gamma(j+1-\alpha)}(1+|\xi|)^{1-\alpha}\left(\frac{d}{d r}\right)^{j} m(r \xi)\right|_{r=2} \\
& +\frac{(-1)^{k}(1+|\xi|)^{1-\alpha}}{\Gamma(k+1-\alpha)} \int_{1}^{2}(s-1)^{k-\alpha}\left(\frac{d}{d s}\right)^{k+1} m(s \xi) d s .
\end{aligned}
$$

By (1), with $\varphi=\varphi_{\xi /|\xi|}$, we have

$$
m(\xi)=\int_{-\infty}^{\infty} e^{-2 \pi i|\xi| u} \varphi(u) d u
$$

hence

$$
\left.\left(\frac{d}{d r}\right)^{j} m(r \xi)\right|_{r=2}=(-2 \pi i|\xi|)^{j} \int_{-\infty}^{\infty} e^{-4 \pi i|\xi| u} u^{j} \varphi(u) d u .
$$

By partial integration this implies

$$
\left.\left(\frac{d}{d r}\right)^{j} m(r \xi)\right|_{r=2}=\frac{1}{2}(-2 \pi i|\xi|)^{j-1} \int_{-\infty}^{\infty} e^{-4 \pi i|\xi| u}\left(u^{j} \varphi\right)^{\prime}(u) d u .
$$

(2) and (15) imply for $0 \leq j \leq N$

$$
\begin{aligned}
\left|\left(\frac{d}{d r}\right)^{j} m(r \xi)\right|_{r=2} \mid & \leq C_{N}|\xi|^{j} \int_{0}^{\infty} u^{j} \varphi(0) e^{-a \varphi(0) u} d u \\
& \leq C_{N} \varphi(0)^{-j}|\xi|^{j} \leq C_{N} \sigma(B)^{j}|\xi|^{j}
\end{aligned}
$$

Moreover, since $\left(u^{j} \varphi\right)^{\prime}(u)=j u^{j-1} \varphi(u)+u^{j} \varphi^{\prime}(u)$, and since $\varphi^{\prime}(u)$ has constant sign for $u \geq 0$ resp. $u \leq 0,(16)$ and (4) yield

$$
\left.\left|\left(\frac{d}{d r}\right)^{j} m(r \xi)\right|_{r=2}\left|\leq C_{N} \varphi(0)^{-(j-1)}\right| \xi\right|^{j-1} \leq C_{N} \sigma(B)^{j-1}|\xi|^{j-1} \text {. }
$$

Together, we obtain

$$
\left.\left|\left(\frac{d}{d r}\right)^{j} m(r \xi)\right|_{r=2}\left|\leq C_{N}(\sigma(B))\right| \xi\right|^{j} /(1+|\xi|),
$$

at least for $j \geq 1$. However, for $j=0,(15)$ and (16) easily imply

$$
|m(\xi)| \leq C(1+\varphi(0)) /(1+|\xi|) \leq C \cdot Q(B) /(1+|\xi|) .
$$


So, together we get

$$
\left|\left(\frac{d}{d r}\right)^{j} m(r \xi)\right|_{r=2} \mid \leq C_{N}(\sigma(B), Q(B)) \frac{|\xi|^{j}}{1+|\xi|}, \quad 0 \leq j<N .
$$

This implies, for $j=0, \ldots, k$,

$$
\left|\frac{(1+|\xi|)^{\alpha-1}}{\Gamma(j+1-\alpha)}\left(\frac{d}{d r}\right)^{j} m(r \xi)\right|_{r=2} \mid \leq C_{N}(\sigma, Q) e^{\pi|\operatorname{Im} \alpha|},
$$

where we made use of the well known asymptotics [8, p. 79]

$$
|\Gamma(x+i y)| \sim e^{-(\pi / 2)|y|}|y|^{(x-1 / 2)} \cdot \sqrt{2 \pi} \quad \text { as }|y| \rightarrow \infty .
$$

So, it remains to estimate the integral term in (13), which, up to the sign, is given by

$$
J(\xi)=\frac{(1+|\xi|)^{1-\alpha}}{\Gamma(k+1-\alpha)}(-2 \pi i|\xi|)^{k+1} \int_{-\infty}^{\infty} F(|\xi| u) u^{k+1} \varphi(u) d u
$$

where

$$
F(t)=\int_{1}^{2}(s-1)^{k-\alpha} e^{-2 \pi i t s} d s .
$$

The estimate of $J(\xi)$ requires more technique, but is essentially based again on (4), so that the rest of the proof of the lemma could be skipped for a first reading. We set

$$
G(u)=\int_{0}^{u} t^{k+1} F(t) d t, \quad u \in \mathbb{R} .
$$

Then

$$
\begin{aligned}
\int_{-\infty}^{\infty} & F(|\xi| u) u^{k+1} \varphi(u) d u \\
& =|\xi|^{-k-2} \int_{-\infty}^{\infty} F(u) u^{k+1} \varphi(u /|\xi|) d u \\
& =-|\xi|^{-k-3} \int_{-\infty}^{\infty} G(u) \varphi^{\prime}(u /|\xi|) d u
\end{aligned}
$$

and hence

$$
\begin{aligned}
|J(\xi)| \leq & C_{N}|\xi|^{-2}(1+|\xi|)^{1-\operatorname{Re} \alpha} \\
& \times\left|\frac{1}{\Gamma(k+1-\alpha)} \int_{-\infty}^{\infty} G(u) \varphi^{\prime}(u /|\xi|) d u\right| .
\end{aligned}
$$


Now

(20)

$$
\begin{aligned}
& \int_{0}^{u} t^{k+1} e^{-2 \pi i t s} d t \\
& =\left(\frac{i}{2 \pi}\right)^{k+1}\left\{(-1)^{k+1}(k+1) ! s^{-(k+2)}\left(e^{-2 \pi i u s}-1\right)\right. \\
& +\sum_{j=0}^{k}\left(\begin{array}{c}
k+1 \\
j
\end{array}\right)(-1)^{j} j !(-2 \pi i)^{k-j} \\
& \left.\times s^{-(j+1)} u^{k+1-j} e^{-2 \pi i u s}\right\}
\end{aligned}
$$

Let

$$
\begin{array}{rl}
G_{j}(u)=u^{k+1-j} \int_{1}^{2}(s-1)^{k-\alpha} s^{-(j+1)} e^{-2 \pi i u s} d s, & \\
j & j=0, \ldots, k+1,
\end{array}
$$

and

$(21)^{\prime} G_{k+2}(u)=G_{k+2}=\int_{1}^{2}(s-1)^{k-\alpha} s^{-(k+2)} d s=\int_{0}^{1} \frac{s^{k-\alpha}}{(s+1)^{k+2}} d s$,

and define for $j=0, \ldots, k+2$

$$
J_{j}(\xi)=\frac{(1+|\xi|)^{1-\operatorname{Re} \alpha}}{|\xi|^{2}}\left|\frac{1}{\Gamma(k+1-\alpha)} \int_{-\infty}^{\infty} G_{j}(u) \varphi^{\prime}(u /|\xi|) d u\right| .
$$

By (20), $G$ is a linear combination of the $G_{j}$, and so it remains only to show that all functions $J_{j}$ have an estimate of the desired type.

For $j=0, \ldots, k+1$,

$$
G_{j}(u)=u^{\alpha-j} e^{-2 \pi i u} \int_{0}^{u} \frac{s^{k-\alpha}}{(1+s / u)^{j+1}} e^{-2 \pi i s} d s,
$$

so Lemma 1 implies for $|u|>1$

$$
\begin{aligned}
G_{j}(u)= & \pm i e^{(\pi / 2)(\alpha-k) i} \Gamma(k+1-\alpha) u^{\alpha-j} e^{-2 \pi i u} \\
& +O\left(e^{(\pi / 2)|\operatorname{Im} \alpha|}|u|^{k-j}\right) .
\end{aligned}
$$

Moreover, if $|u| \leq 1$, then

$$
\begin{aligned}
G_{j}(u)=u^{\alpha-j} \frac{e^{-2 \pi i u}}{k+1-\alpha}\left\{\frac{u^{k+1-\alpha}}{2^{j+1}} e^{-2 \pi i u}\right. & \\
& \left.\quad-\int_{0}^{u} s^{k+1-\alpha} \frac{d}{d s}\left[\frac{e^{-2 \pi i s}}{(1+s / u)^{j+1}}\right] d s\right\},
\end{aligned}
$$


which easily implies

$$
\left|G_{j}(u)\right| \leq C_{N} \frac{|u|^{k+1-j}}{|k+1-\alpha|}, \quad|u| \leq 1 .
$$

(23) and (23)' imply

$$
\begin{aligned}
\left|J_{j}(\xi)\right| \leq & -C_{N} \frac{(1+|\xi|)^{1-\operatorname{Re} \alpha}}{|\xi|^{2}} \\
& \times\left\{\frac{1}{|\Gamma(k+2-\alpha)|} \int_{0}^{1} u^{k+1-j} \varphi^{\prime}(u /|\xi|) d u+e^{(\pi / 2)|\operatorname{Im} \alpha|}\right. \\
& \left.\times \int_{1}^{\infty}\left[u^{\operatorname{Re} \alpha-j}+\frac{u^{k-j}}{|\Gamma(k+1-\alpha)|}\right] \varphi^{\prime}(u /|\xi|) d u\right\} .
\end{aligned}
$$

However, if $j \leq k+1$, then

$$
\left|\int_{0}^{1} u^{k+1-j} \varphi^{\prime}(u /|\xi|) d u\right| \leq-\int_{0}^{1} \varphi^{\prime}(u /|\xi|) d u \leq 2 \varphi(0)|\xi|,
$$

and similarly one shows by (4) that

$$
\begin{aligned}
& \left|\int_{1}^{\infty} u^{\operatorname{Re} \alpha-j} \varphi^{\prime}(u /|\xi|) d u\right|=-|\xi|^{1+\operatorname{Re} \alpha-j} \int_{1 /|\xi|}^{\infty} u^{\operatorname{Re} \alpha-j} \varphi^{\prime}(u) d u \\
& \leq|\xi|^{1+\operatorname{Re} \alpha-j}\left\{|\xi|^{j-\operatorname{Re} \alpha} \varphi(1 /|\xi|)+|\operatorname{Re} \alpha-j| \varphi(0) \int_{1 /|\xi|}^{1} u^{\operatorname{Re} \alpha-j-1} d u\right. \\
& \left.+|\operatorname{Re} \alpha-j| \int_{1}^{\infty} u^{\operatorname{Re} \alpha-j} \varphi(u) d u\right\} \\
& \leq C_{N}(\sigma, Q)|\xi|^{1+\operatorname{Re} \alpha-j}\left(1+|\xi|^{j-\operatorname{Re} \alpha}\right)
\end{aligned}
$$

hence

$$
\left|\int_{1}^{\infty} u^{\operatorname{Re} \alpha-j} \varphi^{\prime}(u /|\xi|) d u\right| \leq \begin{cases}C_{N}(\sigma, Q)|\xi|^{1+\operatorname{Re} \alpha}, & j \leq k, \\ C_{N}(\sigma, Q)|\xi|, & j=k+1 .\end{cases}
$$

Of course $\left|\int_{1}^{\infty} u^{k-j} \varphi^{\prime}(u /|\xi|) d u\right|$ is even dominated by (26). (24), (25) and (26) imply, for $|\xi| \geq 1$,

$$
\left|J_{j}(\xi)\right| \leq C_{N}(\sigma, Q) e^{2 \pi|\operatorname{Im} \alpha|}\left(1+|\xi|^{-\operatorname{Re} \alpha}\right), \quad j=0, \ldots, k+1 .
$$

Moreover, since obviously $\left|G_{k+2}\right| \leq C_{N} /|k+1-\alpha|$, we have $(27)^{\prime} \quad\left|J_{k+2}(\xi)\right| \leq C_{N}|\xi|^{-1-\operatorname{Re} \alpha} \frac{-1}{|\Gamma(k+2-\alpha)|} \int_{0}^{\infty} \varphi^{\prime}(u /|\xi|) d u$

$$
\leq C_{N}(\sigma) e^{\pi|\operatorname{Im} \alpha|}|\xi|^{-\operatorname{Re} \alpha}, \quad|\xi| \geq 1
$$


The last two estimates imply the desired uniform estimates of $m_{\alpha}(\xi)$ and $m_{\alpha}^{\varepsilon}(\xi)$ for $|\xi| \geq 1$.

There remains the case $|\xi|<1$, which is easy: By partial integration

$$
\begin{aligned}
J(\xi)=\frac{(1+|\xi|)^{1-\alpha}}{\Gamma(k+2-\alpha)}\left\{\left.\left(\frac{d}{d s}\right)^{k+1} m(s \xi)\right|_{s=2}\right. & \\
& \left.\quad-\int_{1}^{2}(s-1)^{k+1-\alpha}\left(\frac{d}{d s}\right)^{k+2} m(s \xi) d s\right\}
\end{aligned}
$$

which, together with (15) and (2), implies

$$
|J(\xi)| \leq C_{N}(\sigma, Q) e^{\pi|\operatorname{Im} \alpha|}|\xi|^{k+1} ;
$$

this settles the case $|\xi|<1$.

LEMMA 3. For each unit vector $\eta \in S^{n-1}$ define a distribution $\mu_{\eta}=$ $\partial \chi_{B} / \partial_{\eta}=(\eta \cdot \nabla) \chi_{B}$. Then $\mu_{\eta}$ is even a bounded measure, and

$$
\left\|\mu_{\eta}\right\|_{M\left(\mathbb{R}^{n}\right)}=2 \operatorname{Vol}_{n-1}\left(\pi_{\eta}(B)\right) .
$$

Proof. Let $\varphi \in C_{0}^{\infty}\left(\mathbb{R}^{n}\right)$ with $\|\varphi\|_{\infty}=1$. After rotating coordinates, we may assume that $\eta$ is the $n$th coordinate vector. Writing $\mathbb{R}^{n}=$ $\mathbb{R}^{n-1} \times \mathbb{R}$ with coordinates $(x, u)$, we then have

$$
\left\langle\mu_{\eta}, \varphi\right\rangle=-\int_{B} \frac{\partial \varphi}{\partial \eta}=-\int_{\pi_{\eta}(B)} \int_{B_{x}} \frac{\partial \varphi}{\partial u}(x, u) d u d x,
$$

where $B_{x}$ is the interval $B_{x}=\{u \in \mathbb{R}:(x, u) \in B\}$, with endpoints say $a(x) \leq b(x)$, unless $B_{x}=\varnothing$. So

$$
\left|\left\langle\mu_{\eta}, \varphi\right\rangle\right|=\left|\int_{\pi_{\eta}(B)}[\varphi(b(x))-\varphi(a(x))] d x\right| \leq 2 \operatorname{Vol}_{n-1}\left(\pi_{\eta}(B)\right) ;
$$

hence $\left\|\mu_{\eta}\right\|_{M} \leq 2 \operatorname{Vol}_{n-1}\left(\pi_{\eta}(B)\right)$. Moreover, choosing $\varphi$ to be linear on each section $B_{x}$ such that $\varphi(b(x))=1$ and $\varphi(a(x))=-1$ immediately also gives $\left\|\mu_{\eta}\right\|_{M} \geq 2 \operatorname{Vol}_{n-1}\left(\pi_{\eta}(B)\right)$.

Lemma 4. Let $0<\varepsilon<1 / 2$. Then

$$
\left\|T_{-\varepsilon+i \nu}^{\varepsilon} f\right\|_{p} \leq C_{\varepsilon}(p, \sigma(B), Q(B)) e^{(\pi / 2)|\nu|}\|f\|_{p}, \quad f \in L^{p}\left(\mathbb{R}^{n}\right),
$$

for every $1<p<\infty$.

Proof. Let $\alpha=-\varepsilon+i \nu$. Since

$$
m_{\alpha}^{\varepsilon}(\xi)=-\frac{1}{\Gamma(-\alpha)} \int_{1}^{2}(s-1)^{-\alpha-1}(1+|\xi|)^{1-\varepsilon-\alpha} m(s \xi) d s
$$


it clearly suffices to prove that the multiplier operator corresponding to $(1+|\xi|)^{1-\varepsilon-\alpha} m(s \xi)$ satisfies $(28)$ uniformly for $1 \leq s \leq 2$.

Consider the multiplier $M_{\nu}(\xi)=(1+|\xi|)^{-i \nu}$. This multiplier is of Laplace-transform type in the sense of [8, Ch. II, $\S 4]$, since one easily checks that

$$
\begin{gathered}
(1+\lambda)^{-i \nu}=\lambda \int_{0}^{\infty} a(t) e^{-\lambda t} d t, \quad \lambda \geq 0, \quad \text { where } \\
a(t)=\frac{1}{\Gamma(1+i \nu)}\left[t^{i \nu} e^{-t}+\int_{0}^{t} s^{i \nu} e^{-s} d s\right] .
\end{gathered}
$$

Since $\|a\|_{\infty} \leq C e^{(\pi / 2)|\nu|}$, the general theory of heat-diffusion semigroups [8] implies for $1<p<\infty$

$$
\left\|T_{M_{\nu}} f\right\|_{p} \leq C_{p} e^{(\pi / 2)|\nu|}\|f\|_{p}, \quad f \in L^{p}\left(\mathbb{R}^{n}\right),
$$

where $C_{p}$ is a constant depending only on $p$.

Since $(1+|\xi|)^{1-\varepsilon-\alpha} m(s \xi)=(1+|\xi|)^{-i \nu}(1+|\xi|) m(s \xi)$, and since $\left\|T_{m(s))}\right\|_{p, p}=\left\|T_{m}\right\|_{p, p} \leq|B|=1$ for all $p$, (29) reduces the proof of (28) finally to estimating the multiplier operator corresponding to

$$
m_{0}(\xi)=-2 \pi|\xi| m(\xi)
$$

Define measures $\mu_{j}$ by $\mu_{j}=\partial \chi_{B} / \partial x_{j}, j=1, \ldots, n$. Since

$$
m_{0}(\xi)=\sum_{j=1}^{n}\left(-i \frac{\xi_{j}}{|\xi|}\right)\left(-2 \pi i \xi_{j} m(\xi)\right)
$$

we have

$$
T_{m_{0}} f=\sum_{j=1}^{n} R_{j}\left(\mu_{j} * f\right),
$$

where $R_{j}$ denotes the $j$ th Riesz transform. By a result of Stein [10] (see also [7]), it is known that

$$
\left\|\left(\sum_{j}\left|R_{j} f\right|^{2}\right)^{1 / 2}\right\|_{p} \leq A_{p}\|f\|_{p}, \quad 1<p<\infty,
$$

where $A_{p}$ is independent of $n$. Using a simple duality argument, (31) and (32) imply

$$
\left\|T_{m_{0}} f\right\|_{p} \leq A_{p^{\prime}}\left\|\left(\sum_{J}\left|\mu_{k} * f\right|^{2}\right)^{1 / 2}\right\|_{p}, \quad 1<p<\infty,
$$


where $1 / p+1 / p^{\prime}=1$. Let $g(f)^{2}(x)=\sum_{j}\left|\mu_{j} * f(x)\right|^{2}$. We want to estimate the $L^{p}$-operator norm of the sublinear operator $g$.

If $p=2$, we obtain from (17)

$$
\|g(f)\|_{2}=\left\|T_{m_{0}} f\right\|_{2} \leq\left\|m_{0}\right\|_{\infty}\|f\|_{2} \leq C(\sigma, Q)\|f\|_{2} .
$$

For $p=\infty$, we observe that

$$
|g(f)(x)|=\left|\left(\nabla \chi_{B}\right) * f(x)\right|=\sup _{\eta \in S^{n-1}}\left|\mu_{\eta} * f(x)\right|,
$$

where $\mu_{\eta}$ is defined as in Lemma 4 . This in combination with Lemma 4 implies

$$
\|g(f)\|_{\infty} \leq \sup _{\eta}\left\|\mu_{\eta}\right\|_{M}\|f\|_{\infty}=2 Q(B)\|f\|_{\infty} .
$$

Interpolation between (34) and (36) yields

$$
\|g(f)\|_{p} \leq C(p, \sigma, Q)\|f\|_{p}, \quad 2 \leq p \leq \infty
$$

hence, by (33), also

$$
\left\|T_{m_{0}} f\right\|_{p} \leq C(p, \sigma, Q)\|f\|_{p},
$$

at least for $2 \leq p<\infty$, but by passing to the adjoint operator $T_{m_{0}}^{*}$, we get (37) also for $1<p<2$. This concludes the proof of Lemma 4 .

Proof of Proposition 1. Let $\rho=1-\varepsilon \in$ ]1/2, 1[. From Lemma 2 (ii) and (13) it follows easily that the family $\left\{T_{\alpha}^{\varepsilon}\right\}$ in an admissible family (in the sense of $[12, \mathrm{Ch} . \mathrm{V}]$ ) on every strip $-\varepsilon \leq \operatorname{Re} \alpha \leq N, N>0$. Thus, choosing $N$ sufficiently large and interpolating the estimates in Lemma 2 and Lemma 4 between $\operatorname{Re} \alpha=-\varepsilon$ and $\operatorname{Re} \alpha=N$, we obtain

$$
\left\|T_{1-\varepsilon}^{\varepsilon} f\right\|_{p} \leq C_{\varepsilon}(p, \sigma(B), Q(B))\|f\|_{p}
$$

for any $1<p \leq 2$, hence, by duality, for any $1<p<\infty$. But,

$$
\begin{aligned}
m_{1-\varepsilon}^{\varepsilon}(\xi) & =\left.\left[I^{-\rho} m(r \xi)\right]\right|_{r=1} \\
& =-\frac{1}{\Gamma(\varepsilon)} m(\xi)+\frac{1}{\Gamma(\varepsilon)} \int_{1}^{2}(s-1)^{-\rho} \frac{d m(s \xi)}{d s} d s .
\end{aligned}
$$

Moreover, $(\xi \cdot \nabla)^{\alpha} m(\xi)$ is given by $[5$, p. 51]

$$
(\xi \cdot \nabla)^{\alpha} m(\xi)=\frac{-1}{\Gamma(-\alpha)} \int_{1}^{\infty}(s-1)^{-\alpha-1} m(s \xi) d s \quad \text { if }-1<\alpha<0 .
$$

By partial integration, we see that

$$
(\xi \cdot \nabla)^{\alpha} m(\xi)=\frac{1}{\Gamma(1-\alpha)} \int_{1}^{\infty}(s-1)^{-\alpha} \frac{d m(s \xi)}{d s} d s
$$


for $0<\alpha<1$. A comparison with (39) shows that

$$
\begin{gathered}
(\xi \cdot \nabla)^{\rho} m(\xi)=m_{1-\varepsilon}^{\varepsilon}(\xi)+\frac{1}{\Gamma(\varepsilon)} m(\xi)+\frac{1}{\Gamma(\varepsilon)} \int_{2}^{\infty}(s-1)^{-\rho} \frac{d m(s \xi)}{d s} d s \\
=m_{1-\varepsilon}^{\varepsilon}(\xi)-\frac{1}{\Gamma(-\rho)} \int_{2}^{\infty}(s-1)^{-\rho-1} m(s \xi) d s .
\end{gathered}
$$

Since $\int_{2}^{\infty}(s-1)^{-\rho-1} d s<\infty$, this together with (38) implies

$$
\left\|T_{(\xi \cdot \nabla)^{\rho}} f\right\|_{p} \leq C_{\rho}(p, \sigma, Q)\|f\|_{p} .
$$

3. Examples: The $l^{q}$-unit balls. In the sequel, let $1 \leq q \leq \infty$ be fixed, and let

$$
B_{q}=B_{q}^{n}=\left\{x \in \mathbb{R}^{n}:|x|_{q} \leq 1\right\}
$$

be the unit ball with respect to the $l^{q}$-norm $|x|_{q}=\left(\sum\left|x_{j}\right|^{q}\right)^{1 / q}$ (resp. $|x|_{\infty}=\max \left|x_{j}\right|$, if $\left.q=\infty\right)$.

Let $\kappa(n)=\kappa_{q}(n)$ denote the volume of $B_{q}^{n}$. A straight-forward calculation, using induction on $n$, easily yields $(q<\infty)$

$$
\kappa_{q}(n)=2 \Gamma\left(\frac{1}{q}+1\right)\left[\frac{2}{q} \cdot \Gamma\left(\frac{1}{q}\right)\right]^{n-1} / \Gamma\left(\frac{n}{q}+1\right) .
$$

Choose $m=m_{q}(n)>0$ so, that the body $\widetilde{B}_{q}=m B_{q}$ has volume 1 . (40) implies $m \sim n^{1 / q}$ up to a constant $a_{q}$ (see [4]). Of course, if $q=\infty$, we have $\kappa_{\infty}(n)=2^{n}$, and $m=1 / 2$. Let us determine the constant $L$ mentioned in (5):

Because of the symmetry properties of $\widetilde{B}_{q}$, we have for any $\xi \in S^{n-1}$

$$
\int_{\widetilde{B}_{q}}\langle\xi, x\rangle^{2} d x=\sum_{j} \int_{\widetilde{B}_{q}} \xi_{j}^{2} x_{j}^{2} d x=\left(\sum_{j} \xi_{j}^{2}\right) \int_{\widetilde{B}_{q}} x_{n}^{2} d x=\int_{\widetilde{B}_{q}} x_{n}^{2} d x,
$$

and so we may choose $S\left(B_{q}\right)$ to be $\widetilde{B}_{q}$, and obtain for $L=L\left(B_{q}\right)$

$$
\begin{aligned}
L^{2} & =\int_{\widetilde{B}_{q}} x_{n}^{2} d x=2 \int_{0}^{m} x_{n}^{2}\left(m^{q}-\left|x_{n}\right|^{q}\right)^{(n-1) / q} \kappa_{q}(n-1) d x_{n} \\
& =2 m^{n+2} \kappa_{q}(n-1) \mathrm{B}\left(\frac{3}{q}, \frac{n-1}{q}+1\right),
\end{aligned}
$$

where B denotes the Beta-function.

Since $m^{n} \kappa_{q}(n)=1$, this yields

$$
L^{2}=2 m^{2} \frac{\kappa_{q}(n-1)}{\kappa_{q}(n)} \mathrm{B}\left(\frac{3}{q}, \frac{n-1}{q}+1\right) \sim A_{q}^{2}
$$


by Stirling's formula, and so by (5)

$$
a_{1}^{-1} A_{q} \lesssim \sigma\left(B_{q}^{n}\right) \lesssim a_{1} A_{q},
$$

at least for $q<\infty$. However, for $q=\infty$ clearly $L^{2}=1 / 2$, hence $\sigma\left(B_{\infty}^{n}\right) \approx(2 \sqrt{3})^{-1}$.

In order to estimate $Q\left(\widetilde{B}_{q}^{n}\right)$, we adapt an idea from [4]: Let $\tau$ : $[0, \infty[\rightarrow[0,1]$ be a smooth function satisfying the conditions $(q<\infty)$

$$
\begin{gathered}
\tau=1 \quad \text { on }\left[0, m^{q}\right], \\
\tau=0 \quad \text { on }\left[m^{q}+1, \infty[,\right. \\
-2 \leq \tau^{\prime} \leq 0,
\end{gathered}
$$

and set $K(x)=\tau\left(\sum\left|x_{j}\right|^{q}\right), x \in \mathbb{R}^{n}$. Note that by (42) $\chi_{\widetilde{B}_{q}} \leq K$, and by $(42)^{\prime}\left(m^{q}+1\right)^{1 / q} B_{q} \subset(1+c / n) \widetilde{B}_{q}=\widetilde{\widetilde{B}}_{q}$, hence $\|K\|_{L^{1}} \leq C$. Moreover, we have

$$
\operatorname{Vol}_{n-1}\left(\pi_{\xi}\left(\widetilde{B}_{q}\right)\right)=\frac{1}{2}\left\|\frac{\partial K}{\partial \xi}\right\|_{L^{1}} \quad \text { for all } \xi \in S^{n-1}
$$

This is in fact true if $B=\widetilde{B}_{q}$ is any convex body and $K$ any function which is 1 on $B$, non-increasing with growing distance from $B$, and such that $\partial K / \partial \xi$ is integrable: We may assume without restriction that $\xi=e_{n}$. Then, adapting the notations from the proof of Lemma 4 ,

$$
\begin{aligned}
\int_{B_{x}}\left|\frac{\partial K}{\partial \xi}(x, t)\right| d t & =\int_{b(x)}^{\infty}\left|\frac{\partial K}{\partial t}(x, t)\right| d t+\int_{-\infty}^{a(x)}\left|\frac{\partial K}{\partial t}(x, t)\right| d t \\
& =K(x, b(x))+K(x, a(x))=2
\end{aligned}
$$

hence

$$
\left\|\frac{\partial K}{\partial \xi}\right\|_{L^{1}}=2 \operatorname{Vol}_{n-1}\left(\pi_{\xi}(B)\right) .
$$

In order to estimate $\|\partial K / \partial \xi\|_{L^{1}}$, observe that

$$
\partial K / \partial \xi=q \tau^{\prime}\left(\sum\left|x_{j}\right|^{q}\right) \cdot \sum_{j} \xi_{j} \operatorname{sgn}\left(x_{j}\right)\left|x_{j}\right|^{q-1},
$$

and hence

$$
\begin{array}{r}
\|\partial K / \partial \xi\|_{L^{1}} \leq\left. 2 q \iint_{\widetilde{B}_{q}}\left|\sum_{j} \xi_{j} \operatorname{sgn}\left(x_{j}\right)\right| x_{j}\right|^{q-1} \mid d x \\
=\left.\frac{2 q}{2^{n}} \sum_{\varepsilon_{j}= \pm 1} \int_{\widetilde{B}_{q}}\left|\sum_{j} \varepsilon_{j} \xi_{j} \operatorname{sgn}\left(x_{j}\right)\right| x_{j}\right|^{q-1} \mid d x .
\end{array}
$$


However, Khintchine's inequality

$$
2^{-n} \sum_{\varepsilon_{l}= \pm 1}\left|\sum_{j=1}^{n} \varepsilon_{j} \alpha_{j}\right| \leq C\left(\sum_{j} \alpha_{j}^{2}\right)^{1 / 2}, \quad \alpha_{j} \in \mathbb{R}
$$

implies

$$
\begin{aligned}
\|\partial K / \partial \xi\|_{L^{1}} & \leq C q \int \widetilde{\widetilde{B}}_{q}\left[\sum_{j} \xi_{j}^{2}\left|x_{j}\right|^{2(q-1)}\right]^{1 / 2} d x \\
& \leq C^{\prime} q \cdot\left[\int_{\widetilde{B}_{q}} \sum_{j} \xi_{j}^{2}\left|x_{j}\right|^{2(q-1)} d x\right]^{1 / 2}
\end{aligned}
$$

by Hölder's inequality, since $\operatorname{Vol}_{n}\left(\widetilde{\widetilde{B}}_{q}\right) \leq C$. Because of the symmetry of $\widetilde{\widetilde{B}}_{q}$, this yields

$$
\|\partial K / \partial \xi\|_{L^{1}} \leq C^{\prime \prime} q \cdot\left[\int_{\widetilde{B}_{q}}\left|x_{n}\right|^{2(q-1)} d x\right]^{1 / 2},
$$

and hence, because of (4), (5), (41) and (43),

$$
Q\left(B_{q}^{n}\right) \leq C_{q}, \quad 1 \leq q<\infty,
$$

independently of $n$. So Theorem 1 implies

COROllary 1. Let $1 \leq q<\infty$. Then for all $f \in L^{p}\left(\mathbb{R}^{n}\right)$

$$
\left\|M_{B_{q}^{n}} f\right\|_{p} \leq C(p, q)\|f\|_{p}, \quad 1<p \leq \infty,
$$

independently of $n$.

What can be said about the case $q=\infty$ ?

In this case, an easy geometric consideration shows that for any $\xi \in S^{n-1}$ (see also [1], pp. 41, 45)

$$
\operatorname{Vol}_{n-1}\left(\pi_{\xi}\left(\widetilde{B}_{\infty}\right)\right)=\sum_{F} \operatorname{Vol}_{N-1}(F) \cdot\langle\xi, n(F)\rangle,
$$

where summation is over all faces $F$ of the cube $\widetilde{B}_{\infty}$ whose outward normal $n(F)$ satisfies $\langle\xi, n(F)\rangle \geq 0$. So, if we choose $\xi=$ $n^{-1 / 2}(1,1, \ldots, 1)$, we get

$$
\operatorname{Vol}_{n-1}\left(\pi_{\xi}\left(\widetilde{B}_{\infty}\right)\right)=\sum_{j} \xi_{j}=\sqrt{n}
$$


The same argument easily shows that $\operatorname{Vol}_{n-1}\left(\pi_{\eta}\left(\widetilde{B}_{\infty}\right)\right) \leq \sqrt{n}$ for any $\eta \in S^{n-1}$, and we get

$$
Q\left(B_{\infty}^{n}\right)=\sqrt{n} .
$$

So, our criterion gives a bound for $\left\|M_{B_{\infty}^{n}}\right\|_{p, p}$ which grows with $n$.

Let us conclude with a direct consequence of our results, which appears a bit surprising at the first glance (we do, however, not claim originality for this result). Let $\Sigma\left(\widetilde{B}_{q}^{n}\right)$ denote the surface area of $\widetilde{B}_{q}^{n}$.

CoRollaRy 2. If $1 \leq q<\infty$, then $c \sqrt{n} \leq \Sigma\left(\widetilde{B}_{q}^{n}\right) \leq C q \sqrt{n}$, whereas $\Sigma\left(\widetilde{B}_{\infty}^{n}\right)=2 n$

Proof. By Cauchy's surface formula [1, p. 48]

$$
\begin{aligned}
\Sigma\left(\widetilde{B}_{q}^{n}\right) & =\frac{1}{\kappa_{2}(n-1)} \int_{S^{n-1}} \operatorname{Vol}_{n-1}\left(\pi_{\xi}\left(\widetilde{B}_{q}^{n}\right)\right) d \xi \\
& \leq \frac{\Sigma\left(B_{2}^{n}\right)}{\kappa_{2}(n-1)} Q\left(\widetilde{B}_{q}^{n}\right)=\frac{n \kappa_{2}(n)}{\kappa_{2}(n-1)} Q\left(\widetilde{B}_{q}^{n}\right) ;
\end{aligned}
$$

hence, by (40), (44), for $q<\infty$

$$
\Sigma\left(\widetilde{B}_{q}^{n}\right) \leq C_{q} \sqrt{n} .
$$

Moreover, it is well known [1, p. 104] that the Euclidean ball has minimal surface area among all convex bodies of given volume, and $\Sigma\left(\widetilde{B}_{2}^{n}\right)=m^{n-1} \Sigma\left(B_{2}^{n}\right)=m^{n-1} n \cdot \kappa_{2}(n)=n / m \sim c \cdot \sqrt{n}$ by (40), where $m=m_{2}(n)$. So we also obtain

$$
\Sigma\left(\widetilde{B}_{q}^{n}\right) \geq \Sigma\left(\widetilde{B}_{2}^{n}\right) \sim c \cdot \sqrt{n} .
$$

\section{REFERENCES}

[1] T. Bonnesen and W. Fenchel, Theorie der konvexen Körper, Chelsea Publishing Company (1948).

[2] J. Bourgain, On high dimensional maximal functions associated to convex bodies, Amer. J. Math., 108 (1986), 1467-1476.

[3] _ - On the $L^{p}$-bounds for maximal functions associated to convex bodies in $\mathbb{R}^{n}$, Israel J. Math., 54 (1986), 257-265.

[4] $\longrightarrow$ On dimension free maximal inequalities for convex symmetric bodies in $\mathbb{R}^{n}$, Lecture Notes in Math. 1267, Springer (1987), 168-176. 
[5] A. Carbery, Radial Fourier multipliers and associated maximal functions, Recent Progress in Fourier Analysis, North-Holland Math. Studies, 111 (1985), 49-56.

[6] __ An almost-orthogonality principle with applications to maximal functions associated to convex bodies, Bull. Amer. Math. Soc., 14 No 2 (1986), 269-273.

[7] J. Duoandikoetxea and J. L. Rubio de Francia, Estimations indépendantes de la dimension pour les transformées de Riesz, C. R. Acad. Sc. Paris, t. 300, Série I, no 7 (1985), 193-196.

[8] E. M. Stein, Topics in harmonic analysis, Annals of Math. Studies \#63 (1970), Princeton Univ. Press.

[9] _ The development of square functions in the work of A. Zygmund, Bull. Amer. Math. Soc., 7 (1982), 359-376.

[10] _ Three variations on the theme of maximal functions, Recent Progress in Fourier Analysis, North-Holland Math. Studies 111 (1985), 49-56.

[11] E. M. Stein and J. O. Strömberg, Behavior of maximal functions in $\mathbb{R}^{n}$ for large $n$, Ark. f. Mat., 21 (1983), 259-269.

[12] E. M. Stein and G. Weiss, Introduction to Fourier analysis on Euclidean spaces, Princeton Univ. Press (1971).

Received April 29, 1988.

UNIVERSITÄT BIELEFELD

4800 BIELEFELD 1, FRG 


\section{PACIFIC JOURNAL OF MATHEMATICS EDITORS}

\author{
V. S. VARADARAJAN \\ (Managing Editor) \\ University of California \\ Los Angeles, CA 90024-1555-05 \\ Herbert Clemens \\ University of Utah \\ Salt Lake City, UT 84112 \\ ThOMAs EnRIGHT \\ University of California, San Diego \\ La Jolla, CA 92093
}

R. FINN

Stanford University

Stanford, CA 94305

HeRmanN FlaschKa

University of Arizona

Tucson, AZ 85721

VAUGHAN F. R. Jones

University of California

Berkeley, CA 94720

SteVEn KerckHofF

Stanford University

Stanford, CA 94305

\author{
ROBION KIRBY \\ University of California \\ Berkeley, CA 94720 \\ C. C. MOORE \\ University of California \\ Berkeley, CA 94720 \\ HAROLd StaRK \\ University of California, San Diego \\ La Jolla, CA 92093
}

\section{ASSOCIATE EDITORS}
R. ARENS
E. F. BECKENBACH
B. H. NeUMANN
F. WoLF
K. YoshidA (1906-1982)
(1904-1989)

\section{SUPPORTING INSTITUTIONS}

\section{UNIVERSITY OF ARIZONA UNIVERSITY OF BRITISH COLUMBIA CALIFORNIA INSTITUTE OF TECHNOLOGY UNIVERSITY OF CALIFORNIA MONTANA STATE UNIVERSITY UNIVERSITY OF NEVADA, RENO NEW MEXICO STATE UNIVERSITY OREGON STATE UNIVERSITY}

\author{
UNIVERSITY OF OREGON \\ UNIVERSITY OF SOUTHERN CALIFORNIA \\ STANFORD UNIVERSITY \\ UNIVERSITY OF HAWAII \\ UNIVERSITY OF TOKYO \\ UNIVERSITY OF UTAH \\ WASHINGTON STATE UNIVERSITY \\ UNIVERSITY OF WASHINGTON
}

The Supporting Institutions listed above contribute to the cost of publication of this Journal, but they are not owners or publishers and have no responsibility for its content or policies.

Mathematical papers intended for publication in the Pacific Journal of Mathematics should be in typed form or offset-reproduced (not dittoed), double spaced with large margins. Please do not use built up fractions in the text of the manuscript. However, you may use them in the displayed equations. Underline Greek letters in red, German in green, and script in blue. The first paragraph must be capable of being used separately as a synopsis of the entire paper. In particular it should contain no bibliographic references. Please propose a heading for the odd numbered pages of less than 35 characters. Manuscripts, in triplicate, may be sent to any one of the editors. Please classify according to the scheme of Math. Reviews, Index to Vol. 39. Supply name and address of author to whom proofs should be sent. All other communications should be addressed to the managing editor, or Elaine Barth, University of California, Los Angeles, California 90024-1555-05.

There are page-charges associated with articles appearing in the Pacific Journal of Mathematics. These charges are expected to be paid by the author's University, Government Agency or Company. If the author or authors do not have access to such Institutional support these charges are waived. Single authors will receive 50 free reprints; joint authors will receive a total of 100 free reprints. Additional copies may be obtained at cost in multiples of 50 .

The Pacific Journal of Mathematics is issued monthly as of January 1966. Regular subscription rate: $\$ 190.00$ a year (6 Vols., 12 issues). Special rate: $\$ 95.00$ a year to individual members of supporting institutions.

Subscriptions, orders for numbers issued in the last three calendar years, and changes of address should be sent to Pacific Journal of Mathematics, P.O. Box 969, Carmel Valley, CA 93924, U.S.A. Old back numbers obtainable from Kraus Periodicals Co., Route 100, Millwood, NY 10546.

The Pacific Journal of Mathematics at P.O. Box 969, Carmel Valley, CA 93924 (ISSN 0030-8730) publishes 6 volumes per year. Second-class postage paid at Carmel Valley, California 93924, and additional mailing offices. Postmaster: send address changes to Pacific Journal of Mathematics, P.O. Box 969, Carmel Valley, CA 93924.

\section{PUBLISHED BY PACIFIC JOURNAL OF MATHEMATICS, A NON-PROFIT CORPORATION} Copyright (C) 1990 by Pacific Journal of Mathematics 


\section{Pacific Journal of Mathematics}

Vol. 142, No. $2 \quad$ February, 1990

Christopher J. Bishop, Bounded functions in the little Bloch space . . . . . 209

Lutz Bungart, Piecewise smooth approximations to $q$-plurisubharmonic

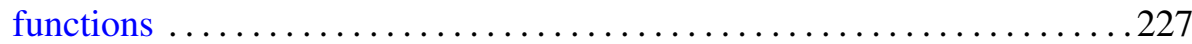

Donald John Charles Bures and Hong Sheng Yin, Outer conjugacy of

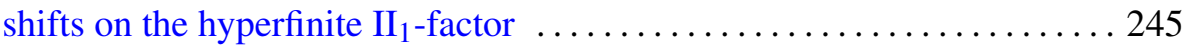

A. D. Raza Choudary, On the resultant hypersurface $\ldots \ldots \ldots \ldots \ldots 259$

Luis A. Cordero and Robert Wolak, Examples of foliations with foliated

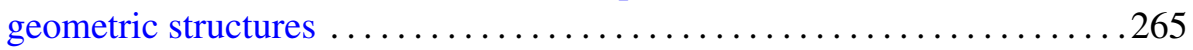

Peter J. Holden, Extension theorems for functions of vanishing mean

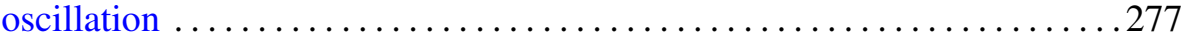

Detlef Müller, A geometric bound for maximal functions associated to convex bodies ........................................ 297

John R. Schulenberger, Time-harmonic solutions of some dissipative problems for Maxwell's equations in a three-dimensional half space . . . 313

Mark Andrew Smith and Barry Turett, Normal structure in Bochner

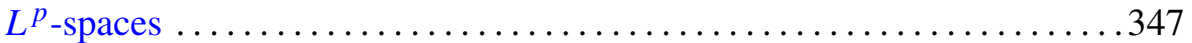

Jun-ichi Tanaka, Blaschke cocycles and generators ................... 357

R. Z. Yeh, Hyperholomorphic functions and higher order partial differential equations in the plane ................................ 379 